\title{
Assisted ventilation for heart failure patients with Cheyne-Stokes respiration
}

\author{
T. Köhnlein*, T. Welte*, L.B. Tan", M.W. Elliott
}

Assisted ventilation for heart failure patients with Cheyne-Stokes respiration. T. Köhnlein, T. Welte, L.B. Tan, M.W. Elliott. (C) ERS Journals Ltd 2002.

ABSTRACT: Patients with chronic congestive cardiac failure (CCF) frequently suffer from central sleep apnoea syndrome (CSAS). Continuous positive airway pressure (CPAP) has been suggested as a treatment. The authors hypothesised that bilevel ventilation might be easier to initiate and superior to CPAP at correcting the sleeprelated abnormality of breathing in patients with $\mathrm{CCF}$.

After excluding those with a history suggestive of obstructive sleep apnoea, 35 patients with CCF (left ventricular ejection fraction $<35 \%$ ) were screened with overnight oximetry and the diagnosis of CSAS was established with polysomnography in 18. Two 14-day cycles of CPAP $(0.85 \mathrm{kPa}(8.5 \mathrm{mbar}))$ or bilevel ventilation $(0.85 / 0.3 \mathrm{kPa}$ $(8.5 / 3$ mbar)) in random order, were compared in a crossover study.

Sixteen patients (13 males), mean age $62.0 \pm 7.4$ yrs completed the study. The pretreatment apnoea/hypopnoea index of $26.7 \pm 10.7$ was significantly reduced by CPAP and bilevel ventilation to $7.7 \pm 5.6$ and $6.5 \pm 6.6$, respectively. The arousal index fell from $31.1 \pm 10.0$ per hour of sleep to $15.7 \pm 5.4$ and $16.4 \pm 6.9$, respectively. Significant and equal improvements with CPAP and bilevel ventilation were found for sleep quality, daytime fatigue, circulation time and New York Heart Association class.

The authors conclude that continuous positive airway pressure and bilevel ventilation equally and effectively improve Cheyne-Stokes respiration in patients with congestive cardiac failure.

Eur Respir J 2002; 20: 934-941.
*Dept of Pulmonary and Intensive Care Medicine, Otto-von-GuerickeUniversität Magdeburg, Magdeburg, Germany. ${ }^{\#}$ Institute for Cardiovascular Research and Dept of Respiratory Medicine, St James's University Hospital Leeds, Leeds, UK.

Correspondence: T. Köhnlein, Ottovon-Guericke-Universität Magdeburg, Zentrum Innere Medizin, Pneumologie und Internistische Intensivmedizin, Leipziger Str. 44, D-39120 Magdeburg, Germany.

Fax: 493916715420

E-mail: thomas.koehnlein@web.de

Keywords: Bilevel ventilation, central sleep apnoea syndrome, Cheyne-Stokes respiration, congestive heart failure, continuous positive airway pressure

Received: December 182001

Accepted after revision: May 132002

T. Köhnlein was funded with a longterm research fellowship from the European Respiratory Society.
Central sleep apnoea syndrome (CSAS) with Cheyne-Stokes respiration (CSR) is common in patients with severe cardiac failure. Up to $45 \%$ of patients with a left ventricular ejection fraction of $\leqslant 40 \%$ may suffer from this breathing disorder during sleep $[1,2]$.

Current concepts of the pathophysiology of CSR suggest it is caused by enhanced carbon dioxide sensitivity, delayed transfer of blood gas tension changes to the chemoreceptors and the influence of adjacent brain centres (circadian rhythms, sleep wake centre) upon the central control of breathing [3]. CSR is characterised by periodic changes of tidal volume, resulting in 30-60-s cycles of hyperventilation, alternating with hypoventilation or apnoea in a crescendo decrescendo pattern. The resumption of respiration from apnoea is associated with arousals and episodes of sympathetic activation. These episodes may occur several hundred times during the night in patients with severe CSAS [4]. The repeated arousals prevent the patient from entering the deeper recuperative stages of sleep, which leads to daytime sleepiness and fatigue. Activation of the sympathetic nervous system coincides with low levels of blood and tissue oxygenation.
Heart rate, blood pressure, and the calculated work load for the heart are periodically increased during sleep. As a consequence, the stress upon the failing heart is multiplied [5]. Sympathetic activation is a well-documented, independent risk factor for progression of cardiac dysfunction and sudden cardiac death [6].

Recent epidemiological studies have shown a significantly worse survival for patients with left heart insufficiency and CSAS [7, 8]. AndREAs et al. [9] concluded that CSR during sleep or wakefulness is associated with mortality or the need for transplantation within a few months. Therefore, treatment of CSR in combination with "optimised" medical therapy may influence the course of chronic heart failure, quality of life and the survival of patients [10].

Continuous positive airway pressure (CPAP) and time-controlled bilevel ventilation (bilevel spontaneous/ timed) are both used as symptomatic approaches to treat sleep disordered breathing, correct hypoxaemia, reduce arousals, decrease sympathetic tone, and improve sleep quality $[11,12]$. However, the application of CPAP to patients with $\mathrm{CHF}$ may be problematic $[13,14]$. CPAP requires a spontaneously 
breathing patient and will be ineffective for central apnoeas, whereas timed bilevel ventilation is able to ventilate a patient even in the absence of spontaneous respiratory effort. Furthermore, some patients find breathing out against positive pressure uncomfortable. Bilevel ventilation has been suggested as an alternative, but little is known about the efficacy or tolerance of bilevel ventilation in these patients and, in particular, it has not been compared to CPAP. The authors hypothesised that bilevel ventilation might be more effective than CPAP in terms of reducing pathological respiratory events and be better tolerated by patients.

The aim of this prospective randomised, nonblinded, crossover, clinical trial was to compare the effect of CPAP and bilevel ventilation on the ventilatory pattern during sleep in a cohort of patients with CSAS during sleep. Secondary outcome parameters were the effect on blood oxygen saturation, arousals from sleep, sleep quality, circulation time, quality of life, daytime fatigue, and New York Heart Association (NYHA) functional class.

\section{Patients and methods}

\section{Subjects}

Successive patients of both sexes, aged 18-80 yrs, were screened in a cardiac outpatient clinic at the Leeds Teaching Hospitals National Health Service (NHS) Trust (Leeds, UK) between April and November 2000.

Patients with a $>1$ yr history of chronic left heart failure, in NYHA functional class II and III due to ischaemic heart disease or dilated cardiomyopathy, qualified for the study. Furthermore, inclusion criteria were a left ventricular ejection fraction of $\leqslant 35 \%$. The subjects had to be outpatients in stable clinical condition over the last 3 months before study entry and their medical treatment was optimised, based on the guidelines of the European Society of Cardiology [15]. Only minor changes in the dosage of diuretics, but no introduction or withdrawal of any other medication with an effect on the circulation or control of breathing, were allowed in the 3 weeks prior to study entry and during participation in the study. The cardiologic assessment was provided by an independent cardiologist (L.B. Tan) who was blinded for the course of the study.

Exclusion criteria included any pulmonary or neurological disease that might affect ventilation or the control of breathing (such as central alveolar hypoventilation syndrome, chronic obstructive pulmonary disease, pulmonary fibrosis, chronic infections in the respiratory tract, previous stroke, transient ischaemic attack, neuropathy and tumour). Further exclusion criteria were myocardial infarction within 6 months of study entry, severe ventricular arrhythmia, atrial fibrillation [16], cardiac valve replacement, current smoking, known or a strong clinical suspicion of obstructive sleep apnoea ( $>10$ obstructive apnoeas per hour of sleep or $>10 \%$ of all detected apnoeas being obstructive in nature), diabetes mellitus, and (suspicion of) alcohol or drug abuse. Patients with any noncardiologic medication that would have a possible influence on the control of breathing (e.g. sedatives, tranquillisers, theophylline) were also excluded.

Any deterioration in the subject's condition as a result of participation in this study, judged by clinical assessment, polysomnography or from the patient's own impression, warranted immediate withdrawal from the study.

The study was approved by the Local Research Ethics Committee of the Leeds Teaching Hospitals NHS Trust. Informed written consent was obtained from every patient prior to study entry.

\section{Experimental protocol}

The procedure for identifying patients with CSAS consisted of two steps. First, potential patients were provided with a miniature oxygen saturation record-

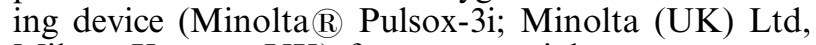
Milton Keynes, UK) for an overnight measurement at home. This recording was interpreted according to the study of STANIFORTH et al. [17]. If the oxygen saturation recording showed prolonged sections with regular fluctuations in oxygen saturation of $>4 \%$ and if the percentage of the night $(23-6 \mathrm{~h})$ spent with an oxygen saturation of $\leqslant 90 \%$ was $>5 \%$, the recording was suggestive of nocturnal breathing abnormalities. Subjects with these findings underwent screening polysomnography in the sleep laboratory to establish or exclude the diagnosis of central sleep apnoea and CSR (see below for criteria).

CSR patients were randomised and entered a crossover study design (fig. 1). The randomisation procedure was performed with closed envelopes containing a label for the first treatment mode. The interventions were treatment cycles with CPAP or bilevel ventilation, with each cycle consisting of 12

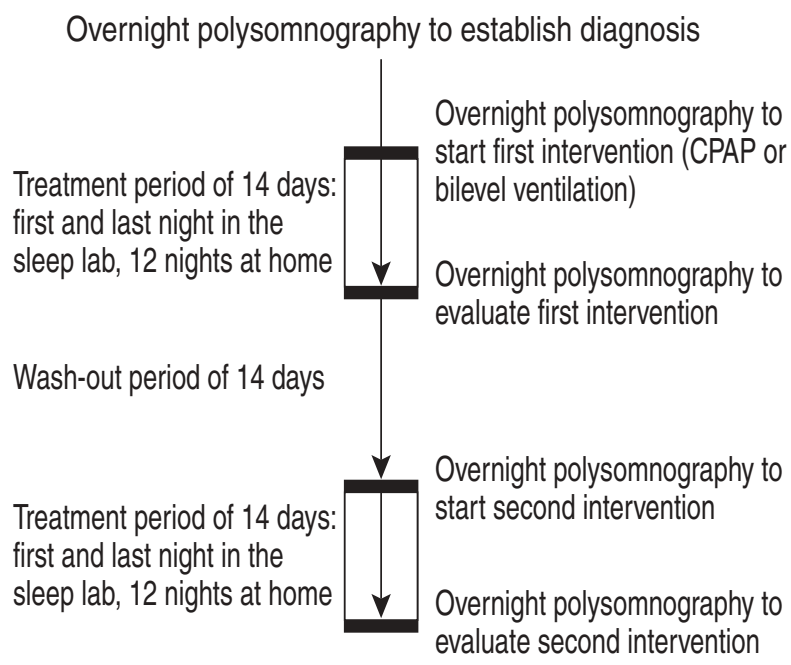

Fig. 1.-Two 14-day treatment cycles, separated by a 14-day washout period, were provided. Patients were assigned randomly to continuous positive airway pressure (CPAP) or bilevel ventilation as first treatment. The other intervention was performed in the second treatment cycle. 
treatment nights at home. Polysomnography was performed at the beginning and at the end of each treatment cycle. Both cycles were separated by a 14-day washout period.

The polysomnographic studies at the beginning of each treatment cycle were performed to confirm the tolerance to the intervention and to exclude detrimental effects of CPAP or bilevel ventilation. Data for analysis in this study were obtained from the first polysomnography during spontaneous breathing and from the polysomnographies at the end of each treatment cycle.

\section{Polysomnography}

Full polysomnography with simultaneous recording of electroencephalography (EEG) (C3A2; C4A1), eye movements, submental electromyography (EMG), airflow with a nose/mouth thermistor, respiratory efforts of the chest and abdomen (inductance plethysmography), oxygen saturation, body position, electrocardiography (ECG), heart beat-to-beat interval, respiratory sound, mask air flow and mask pressure, was performed with an Alice 4 system (software version 1.7.16; Respironics Inc., Pittsburgh, PA, USA). Polysomnography was performed according to the standards of the American Thoracic Society [18] and scored according to the criteria of RECHTSCHAFFEN and KALES [19]. The typical ventilatory pattern of CSR, with regular fluctuation of tidal volume, was identified visually. All scoring was performed by the same investigator (T. Köhnlein). It was not possible to blind either the patients or the investigator to the type of device used.

Circulation time was calculated as described in detail elsewhere [20]. In brief, 15 randomly selected sequences with periodic breathing in sleep stage II were analysed for the time interval between the resumption of respiratory efforts and the nadir of the oxygen saturation at the finger tip of the index finger of the left hand (fig. 2). Assessment of the circulation time was performed in high-resolution printouts with oxygen saturation recording in 1-s intervals.

\section{Symptom assessment}

Daytime sleepiness and fatigue were quantified with the Epworth sleepiness scale [21]. Health status was assessed with the short-form health survey (SF)-36 quality-of-life questionnaire [22], a generic questionnaire, which has been used previously for evaluation of patients with chronic cardiac disease and obstructive sleep apnoea [23].

\section{Continuous positive airway pressure and noninvasive ventilation}

Nasal CPAP (nCPAP) was delivered with a Sullivan V flow generator (Resmed Inc., Poway, CA, USA). All patients, independent of age, body mass index (BMI) or sex, were treated with $0.85 \mathrm{kPa}(8.5 \mathrm{mbar})$ mask pressure. Bilevel ventilation was performed with a VPAP II flow generator (Resmed Inc.) in the spontaneous-timed mode: during spontaneous breathing, subjects were ventilated in assisted mode with fixed pressure levels of $0.85 \mathrm{kPa}(8.5 \mathrm{mbar})$ during inspiration and $0.3 \mathrm{kPa}$ (3 mbar) during expiration. In case of an apnoea, the ventilator switched to controlled (timed) ventilation after a latency of $5 \mathrm{~s}$. During controlled ventilation, the breathing frequency was fixed at $12 \mathrm{breaths} \cdot \mathrm{min}^{-1}$, and the applied pressures were the same as during assisted ventilation. Controlled ventilation lasted until sufficient spontaneous breathing to trigger the assisted ventilation mode reappeared [24].

The run time of all ventilators was recorded with an hour meter in the device recording the actual time the patient received assisted ventilation within the last fourteen 24-h cycles (not visible to the patient). Ultra Mirage masks (Resmed Inc.) or Respironics Special nasal masks (Respironics Inc.) were used according to the subject's preference.

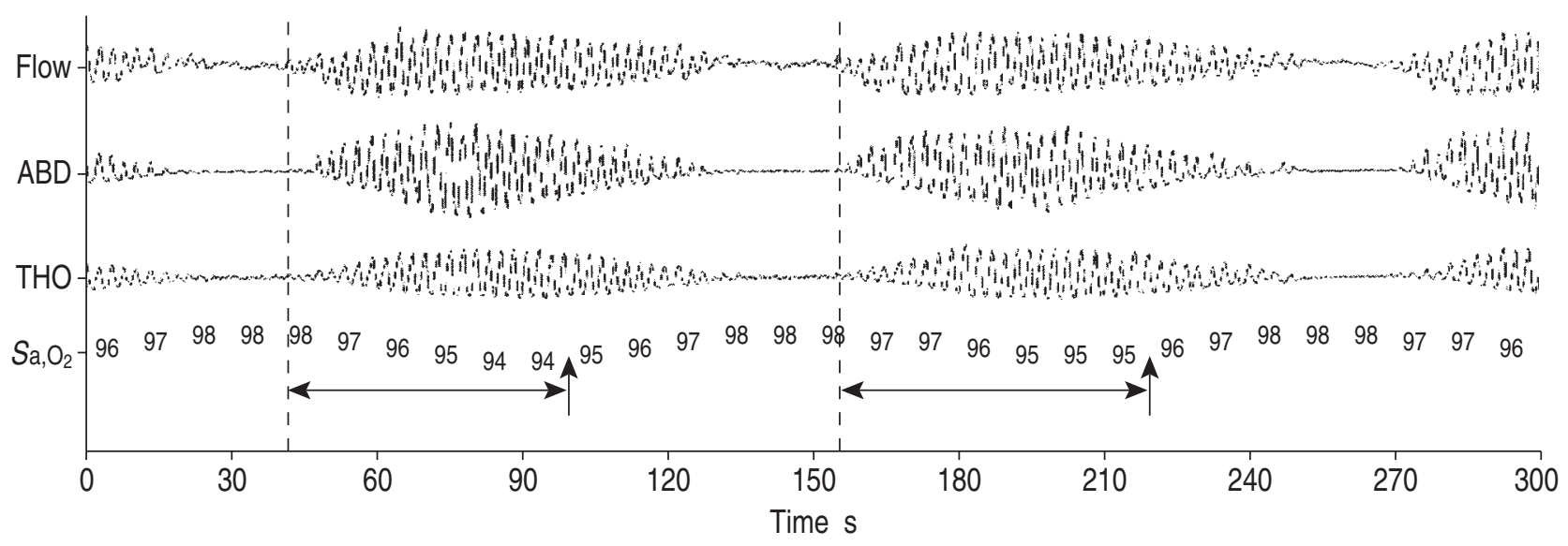

Fig. 2. - Simultaneous recording of ventilation and oxygen saturation $\left(\mathrm{S}_{\mathrm{a}}, \mathrm{O}_{2}\right)$ during polysomnography. Typical pattern of Cheyne-Stokes respiration with regular fluctuation of tidal volume. Circulation time (double headed arrow) is defined as the interval between the resumption of ventilation (--) and the nadir of $\mathrm{Sa}_{\mathrm{a}} \mathrm{O}_{2}$ at the finger tip of the index finger of the left hand (single headed arrow). Flow: oro-nasal air flow; ABD: abdominal ventilatory effort; THO: thoracic ventilatory effort. 


\section{Data analysis}

All variables are given as mean \pm SEM. All data were analysed for a normal distribution with the Kolmogorov-Smirnov test. One-way repeated measures analysis of variance (ANOVA) was applied for all parametric data. A p-value of $\leqslant 0.05$ was regarded as statistically significant.

\section{Results}

A total of 330 chronic heart failure patients were considered for this study. Thirty five patients, meeting all inclusion and exclusion criteria were screened with overnight pulse-oximetry and $18(51 \%)$ were found to have CSR. The diagnosis of central sleep apnoea and CSR was established, obstructive sleep apnoea excluded, with full polysomnography in all subjects. Two subjects withdrew after the first intervention night (CPAP in both cases) because of the inconvenience of the procedure. Sixteen patients (13 males) complied with all aspects of the protocol and completed the study. The mean age was $62.0 \pm 7.4$ yrs (range 49-77) and the mean BMI was $27.3 \pm 3.2 \mathrm{~kg} \cdot \mathrm{m}^{-2}$ (range 20.7-32.3). The body weight of all subjects remained stable within $\pm 1.5 \mathrm{~kg}$ throughout the study. All patients suffered from advanced left ventricular insufficiency; the mean left ventricular ejection fraction was $23.6 \pm 6.9 \%$ (range 15-35). Eleven patients had severe coronary artery disease, with five of them having had coronary artery bypass surgery in the past. The remaining five subjects had severe, idiopathic dilated cardiomyopathy. At study entry, four subjects were in NYHA functional class II, and twelve in class III (table 1). Their medical treatment was unchanged throughout the study, according to the criteria described above. Ten subjects were randomised to have CPAP as their first treatment.

The initial polysomnography revealed CSR in all subjects, averaging $37.1 \pm 16.6 \%$ of the total sleep time
(TST). CSR was typically found in sleep stages I, II and occasionally during rapid eye movement (REM) sleep. In nearly all subjects the breathing pattern was unaffected during slow wave sleep (SWS). In one subject (no. 2), CSR was documented during wakefulness shortly before the onset of sleep. Obstructive respiratory events were rare, and in the whole study population the amount of snoring ranged $0-4 \%$ of the TST.

After 2 weeks of treatment, both CPAP and bilevel ventilation reduced CSR significantly from $37.1 \pm$ $16.6 \%$ to $14.1 \pm 17.5 \% \quad(\mathrm{p}<0.001)$ and $13.8 \pm 17.6 \%$ $(\mathrm{p}<0.001)$ of TST, respectively (fig. 3a). The high amount of CSR was reflected in a pretreatment apnoea/hypopnoea index (AHI) of $26.7 \pm 10.7$ (range 15-46), which was significantly reduced by CPAP and bilevel ventilation to $7.7 \pm 5.6(\mathrm{p}<0.00)$ and $6.5 \pm 6.6$ $(\mathrm{p}<0.00$, respectively (fig. $3 \mathrm{~b})$ ). There were no significant differences in any of the measured variables between CPAP and bilevel ventilation, as they both markedly improved sleep disordered breathing.

The average oxygen saturation was normal for all subjects across the night $(94.4 \pm 1.8 \%)$, and did not change significantly with CPAP $(94.6 \pm 1.9 \%, \mathrm{p}=0.22)$ or bilevel ventilation $(94.7 \pm 1.6 \%, \mathrm{p}=0.645)$. However, in association with apnoeas and hypopnoeas, severe, short-term oxygen desaturation was documented. Before treatment, the total time spent with oxygen saturation $<90 \%$ during sleep was $18.1 \pm 30.9 \mathrm{~min}$, and this was significantly reduced with CPAP to $1.6 \pm$ $1.9 \min (\mathrm{p}<0.029)$ and with bilevel ventilation to $0.8 \pm 1.1 \mathrm{~min}(\mathrm{p}=0.039$ ) (fig. $3 \mathrm{c}$ ). In parallel with the improvements in ventilation, the amount of SWS and REM sleep returned to the normal range (fig. $4 \mathrm{a}$ and b). The total frequency of arousals was increased before treatment (arousal index $31.1 \pm 10.0 \cdot \mathrm{h}^{-1}$ ) and was significantly reduced by both CPAP $\left(15.7 \pm 5.4 \cdot \mathrm{h}^{-1}\right.$, $\mathrm{p}<0.001)$ and bilevel ventilation $\left(16.4 \pm 6.9 \cdot \mathrm{h}^{-1}, \mathrm{p}<0.001\right)$ (fig. 4c).

The influence of both interventions on haemodynamic parameters was assessed by calculating the

Table 1. - Anthrophometric data

\begin{tabular}{|c|c|c|c|c|c|}
\hline Subject no. & Age yrs & $\mathrm{BMI} \mathrm{kg} \cdot \mathrm{m}^{-2}$ & NYHA class & Ejection fraction $\%$ & Medication \\
\hline 1 & 53 & 22.4 & 3 & 35 & $\mathrm{AI}, \mathrm{BB}, \mathrm{DIG}, \mathrm{DIU}, \mathrm{N}$ \\
\hline 2 & 70 & 20.7 & 3 & 15 & AI, DIG, DIU, N \\
\hline 3 & 53 & 27.7 & 3 & 20 & AI, DIG, DIU, N \\
\hline 4 & 69 & 27.0 & 3 & 15 & AI, DIG, DIU, N \\
\hline 5 & 66 & 26.6 & 3 & 20 & AI, BB, DIU, N \\
\hline 6 & 64 & 28.7 & 3 & 25 & BB, DIU, N \\
\hline 7 & 59 & 28.8 & 3 & 30 & $\mathrm{AI}, \mathrm{BB}, \mathrm{DIG}, \mathrm{DIU}, \mathrm{N}$ \\
\hline 8 & 57 & 29.4 & 3 & 20 & AI, DIU, N \\
\hline 9 & 72 & 30.5 & 2 & 25 & $\mathrm{AI}, \mathrm{BB}, \mathrm{N}$ \\
\hline 10 & 56 & 29.1 & 3 & 30 & $\mathrm{AI}, \mathrm{BB}, \mathrm{DIU}, \mathrm{N}$ \\
\hline 11 & 61 & 28.1 & 3 & 20 & AI, DIU, N, CCB \\
\hline 12 & 49 & 30.5 & 2 & 25 & AI, DIU, DIG \\
\hline 13 & 59 & 25.0 & 2 & 30 & $\mathrm{AI}, \mathrm{BB}$ \\
\hline 14 & 66 & 32.3 & 2 & 20 & AI, BB \\
\hline 15 & 77 & 21.9 & 3 & 15 & AI, DIU, N \\
\hline 16 & 61 & 27.5 & 3 & 35 & AI, DIU, CCB \\
\hline Mean \pm SD & $62.0 \pm 7.4$ & $27.3 \pm 3.2$ & $2.8 \pm 0.4$ & $23.8 \pm 6.5$ & \\
\hline
\end{tabular}

BMI: body mass index; NYHA: New York Heart Association; AI: angiotensin-converting enzyme inhibitor; BB: $\beta$-blocker; DIG: digoxine; DIU: diuretics; N: nitrate; $\mathrm{CCB}$ : calcium channel blocker. 

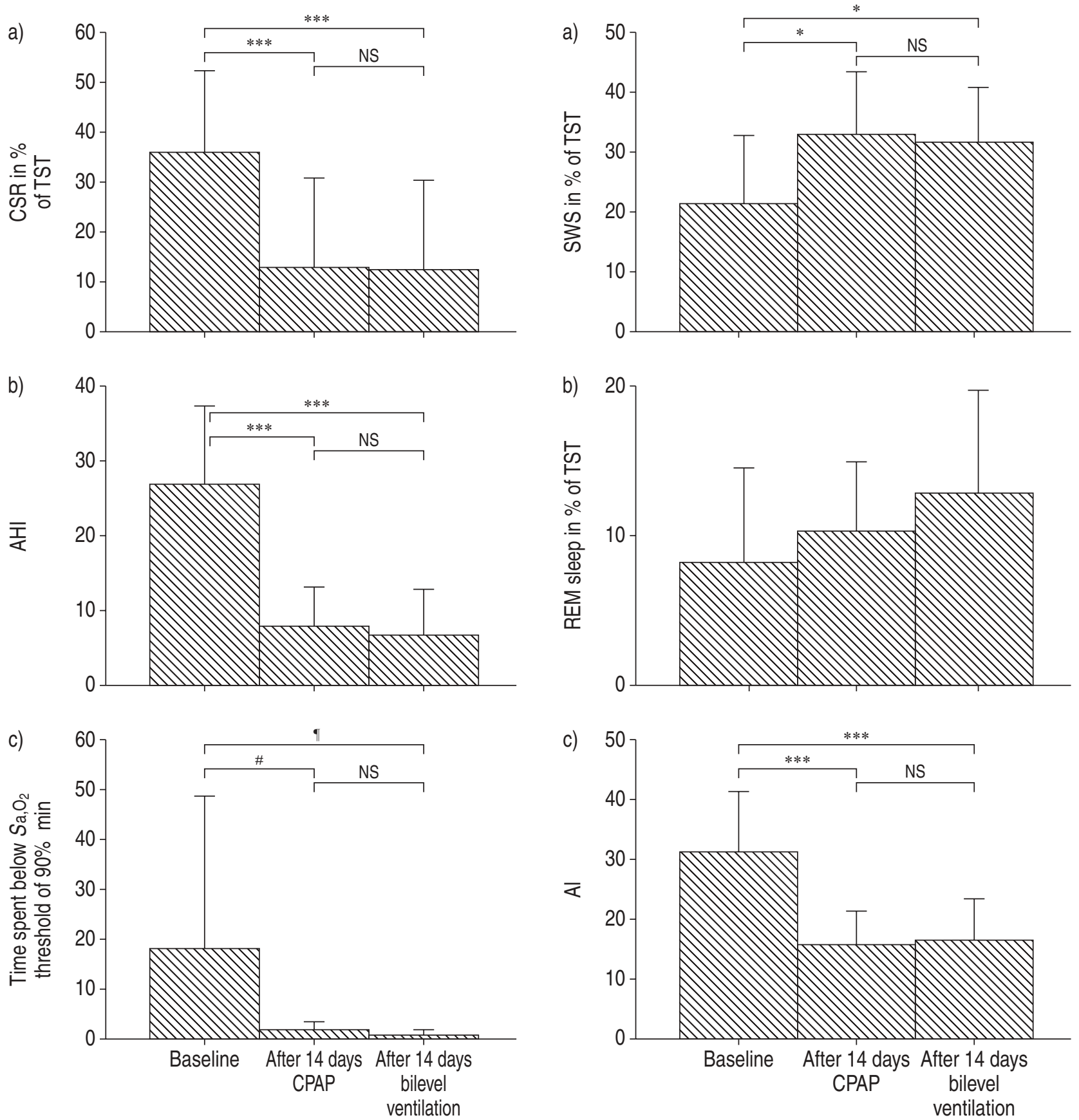

Fig. 3.-Percentage of a) Cheyne-Stokes respiration (CSR) in total sleep time (TST), b) apnoea/hypopnoea index (AHI) and c) time spent below the critical oxygen saturation $\left(\mathrm{Sa}_{\mathrm{a}} \mathrm{O}_{2}\right)$ threshold of $90 \%$ during sleep before and after treatment. NS: nonsignificant. $* * *: \mathrm{p}<0.001 ;{ }^{\#}: \mathrm{p}=0.029 ;{ }^{\uparrow}: \mathrm{p}=0.039$.

average circulation time between the lungs and the left index fingertip. Before treatment the mean circulation time was $35.8 \pm 6.1 \mathrm{~s}$, which decreased to $29.8 \pm 6.1 \mathrm{~s}$ $(\mathrm{p}<0.001)$ with CPAP and to $29.9 \pm 5.3 \mathrm{~s} \quad(\mathrm{p}<0.001)$ with bilevel ventilation (fig. 5).

The average NYHA functional class for all subjects was estimated at $2.8 \pm 0.4$ before treatment. After 14 days, the NYHA functional class had improved to $2.0 \pm 0.4 \quad(\mathrm{p}<0.003)$ with $\mathrm{CPAP}$ and to $1.9 \pm 0.5$

Fig. 4.-a) Percentage of slow wave sleep (SWS) and b) rapid-eye movement (REM) sleep as percentage of total sleep time (TST), and c) arousal index (AI) before and after treatment. NS: nonsignificant. *: $\mathrm{p}<0.05 ; * * *: \mathrm{p}<0.001$.

$(\mathrm{p}<0.001)$ with bilevel ventilation. The mean Epworth sleepiness score before treatment was $12.3 \pm 2.6$. This marker of daytime sleepiness was significantly reduced to $7.5 \pm 2.4 \quad(\mathrm{p}<0.001)$ and $7.1 \pm 2.5 \quad(\mathrm{p}<0.001)$ after CPAP or bilevel therapy, respectively. Health status, as assessed by the SF-36 questionnaire, showed a trend towards improvements in physical health and emotional health as well as in the categories of physical and social activity, but these did not reach statistical significance (fig. 6). No participant scored 


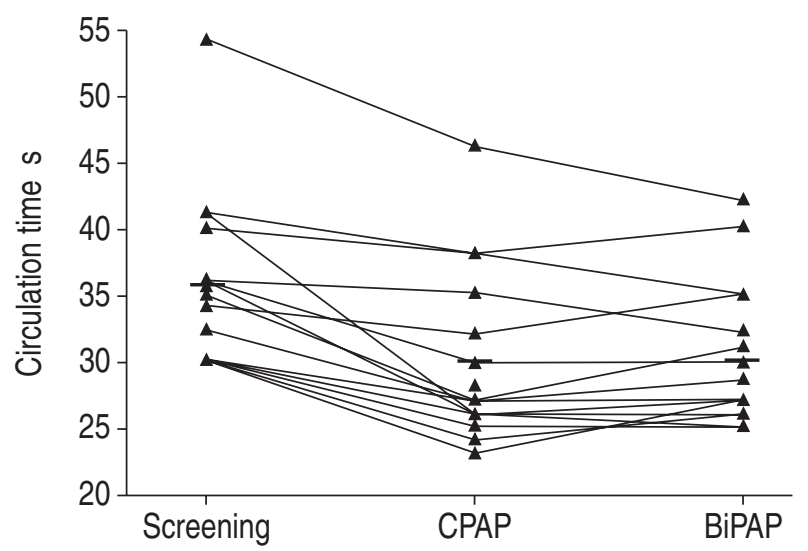

Fig. 5.-Circulation time before treatment and during continuous positive pressure ventilation (CPAP) or bilevel positive airway pressure ventilation (BiPAP). -: mean values.

worse in any item of the test after treatment. During the 14-day treatment periods, the mean daily ventilator run time was $5.6 \pm 1.5 \mathrm{~h}$ for CPAP and $5.1 \pm 1.5 \mathrm{~h}$ for bilevel ventilation ( $\mathrm{p}=$ nonsignificant $(\mathrm{NS})$ ).

\section{Discussion}

In agreement with other reports [11, 14, 25, 26], the authors found that CPAP is an effective treatment for patients with CSR. This was manifested by significant improvements in the percentage of CSR during sleep, AHI, sleep quality, arousal index, mean circulation time, Epworth sleepiness score and the NYHA class. These results with CPAP are similar to previous studies in patients with heart failure. The reduction of hypopnoeas and apnoeas with CPAP was similar to that found in the work of NAUGHTON et al. [27] and JAVAHERI [28]. JAVAHERI [28] also measured arousals and oxygenation before and after CPAP treatment, and the author's responders to CPAP showed similar results to those observed in the subjects present in this study. The authors did not find that the more complicated and more expensive bilevel ventilation conferred any advantage.

The finding that CPAP was well tolerated was surprising, since it has not been experienced by the authors in routine clinical practice. Only two subjects were unable to tolerate noninvasive positive pressure in this study, both withdrawing after 1 night of treatment with CPAP. The remaining 16 patients managed to fulfil all requirements of the protocol.

There was not a high proportion of nonresponders to CPAP, as described by JAVAHERI [28], or bilevel ventilation. However, the baseline AHI in the patients in the current study (26.7 \pm 10.7 , range 15-46) was considerably lower than in the total cohort $(51 \pm 23)$ in the study by JAVAHERI [28], but was close to the mean AHI of responders to CPAP $(36 \pm 15)$ in the latter study [28].

Similar effects of bilevel ventilation on CSA were demonstrated by TESCHLER et al. [29], who investigated four different treatment modes (oxygen, CPAP, bilevel ventilation and adaptive pressure support

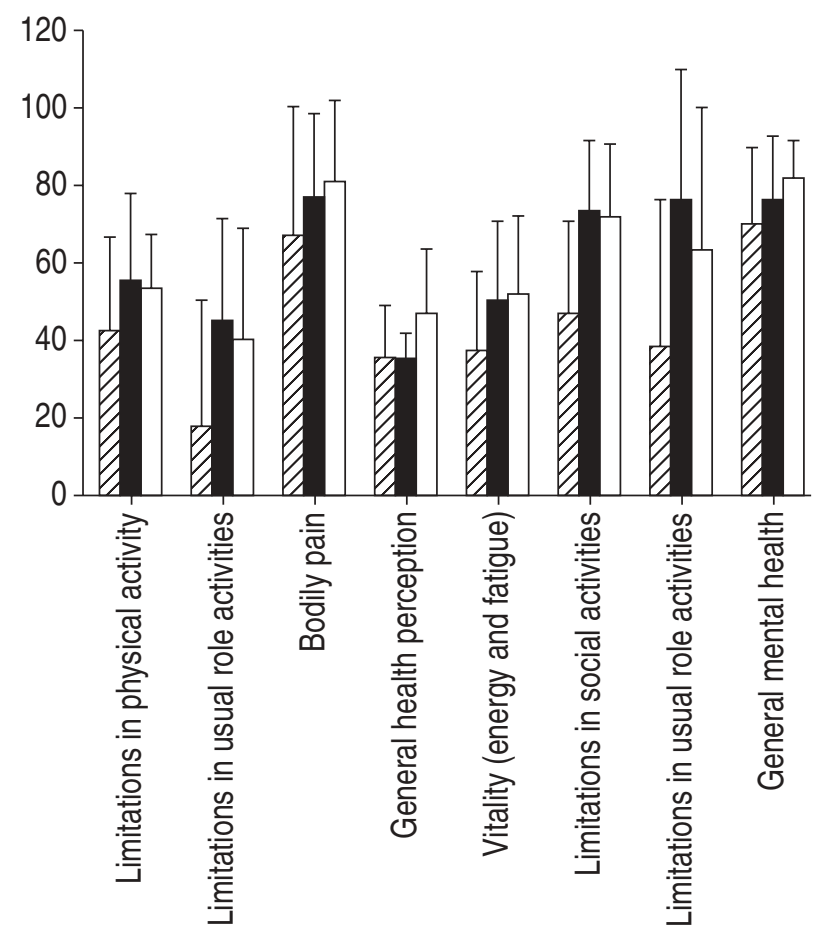

Fig. 6.-Evaluation of quality of life with the short-form health survey (SF)-36 questionnaire before $(\mathbb{Q})$ and after 14 days of treatment with either continuous positive airway pressure (CPAP, $\square$ ) or bilevel positive airway pressure ventilation (BiPAP, $\square$ ). The scales range $0-100$ (0: bad quality of life; 100: best possible health status). All patients improved in all items after CPAP or BiPAP, but this did not reach statistical significance.

servo-ventilation) during 1 treatment night each. In contrast to the present study, TESCHLER et al. [29] did not find that CPAP was effective. The current study evaluated each treatment after a 12-day treatment period at home to rule out the influence of adaptation problems and first night effects on the assessment of the treatment mode.

The daily ventilator use was satisfactory for both interventions. The run time for CPAP and bilevel ventilation exceeded the average daily run time from the authors' laboratory for patients with obstructive sleep apnoea syndrome $\left(4.3 \mathrm{~h} \cdot \mathrm{day}^{-1}\right)$. The involvement of a full time doctor in the study might have resulted in greater involvement with the patient than would be seen in normal every day clinical practice and the relatively short period of 14 days might also have been a factor.

The choice of pressure settings was guided by the possible impact of increased intrathoracic pressure on the cardiovascular system and this may have been a further reason for the good compliance with CPAP in this study. BuCKLE et al. [14] found that their congestive heart failure patients did not tolerate CPAP pressures of $>0.5-0.75 \mathrm{kPa}$ (5-7.5 mbar). In this study, relatively low pressures of $0.85 \mathrm{kPa}$ (8.5 mbar) and $0.85-0.3 \mathrm{kPa}(8.5 / 3 \mathrm{mbar})$ were chosen for CPAP or bilevel ventilation, respectively, similar to those of NAUGHTON et al. [11], who used pressures from $0.5-1.25 \mathrm{kPa}$ (5 to $12.5 \mathrm{mbar}$ ), because of the observation of BECKER et al. [30] that there was a 
decrease in cardiac output with CPAP of $>1 \mathrm{kPa}$ (10 mbar) or with bilevel ventilation of $\geqslant 1 / 0.5 \mathrm{kPa}$ (10/5 mbar) in patients with sleep apnoea.

The applied pressures in this study were effective in all subjects, regardless of the severity of CSR, their BMI or body constitution. Treatment of CSAS does not seem to be critically dependent on the applied pressure, in contrast to patients with obstructive sleep apnoea syndrome [31]. The fact that ventilatory parameters during sleep were improved in all patients with the same settings suggests that the beneficial effects of positive intrathoracic pressure upon the failing heart are the predominant mechanism of benefit. In various studies, BRADLEY and co-workers $[11,26]$ demonstrated that CPAP is effective not only in improving respiratory events but also in reducing cardiac pre and after load, transmural pressure of intrathoracic blood vessels [32] and cardiac dimensions [33]. The current finding of a reduction in circulation time is also consistent with improved cardiac performance.

There were no adverse effects of assisted ventilation in the patients in this study. Hyperventilation is a major aspect of the pathophysiology of CSAS in chronic heart failure patients. CPAP and bilevel ventilation might further increase hyperventilation by reducing the work of breathing. Although ventilation was assessed by visual analysis of the ventilation signals in the polysomnography recordings only, hyperventilation was not detected with any intervention in any patient. Ventilation was regularised with CPAP and bilevel ventilation, i.e. the pre-existing pathological breathing pattern was improved in all subjects. This was true for the relatively low pressure settings in this study. In view of the satisfactory results, higher ventilatory pressures were not studied, and therefore no information on the effect of ventilatory pressure was obtained.

Carbon dioxide tensions were not measured. Direct measurement is not feasible during sleep unless an arterial line is available, but this was not performed with respect to patient safety and comfort. Transcutaneous measurement techniques are not sufficiently responsive for patients with rapid alterations of their breathing pattern and the measurement of gas samples in the exhaled air stream in the nose is quick, but during CPAP or bilevel ventilation there is no undisturbed exhaled air stream for analysis [34]. Assisted ventilation could theoretically worsen CSR by reducing the carbon dioxide tension in arterial blood below the apnoeic threshold. The fact that an improvement in CSR was found suggests that this did not occur even though carbon dioxide tensions were not measured. This was probably because of the low pressures that were chosen and higher pressures may have had a deleterious effect on CSR through this mechanism.

The lack of a placebo limb is a potential limitation of this study. However, in other studies, CPAP has been shown to have a beneficial effect on CSR, but because implementation may be difficult, the primary interest was to compare CPAP and bilevel ventilation in CHF patients, and the addition of a placebo limb would have considerably added to the complexity of the protocol for the patients. This study does support the observation in other studies of a beneficial effect of assisted ventilation, even though it was not the primary aim of this study. One advantage of this study compared to previous work is that the selection of subjects was guided by restrictive inclusion and exclusion criteria to avoid the confounding effects of comorbidity. Previous studies with similar design [11, 28, 35] might have been biased, because they were not controlled for cofounders influencing the control of breathing, such as medication and stroke, or the underlying cardiac rhythm.

Health status showed a trend towards improvement and no patient became worse with positive pressure ventilation. The generic questionnaire, SF-36, is probably not sensitive enough to detect changes that might be picked up with disease-specific questionnaires. The improvement in the NYHA functional class of many patients is a further indicator of improved physical health under either treatment. However, the estimation of the NYHA class by the investigators is subjective and therefore open to bias.

In conclusion, this randomised, crossover, open clinical trial confirmed the finding of other authors of significant benefits of continuous positive airway pressure and bilevel ventilation in patients with stable chronic heart failure due to ischaemic heart disease or idiopathic cardiomyopathy and Cheyne-Stokes respiration. However, in the absence of a control group, the fact that this might have been because of other factors, such as closer observation, cannot be excluded. Continuous positive airway pressure and bilevel ventilation with low pressures were equally effective in improving sleep quality, blood oxygenation, arousals from sleep and daytime symptoms. Both interventions were well accepted over the 14-day treatment periods and these data do not suggest a role for bilevel ventilation, the equipment for which is more expensive than that for continuous positive airway pressure. Low pressures are effective and well tolerated. The impact of treatment on the pathophysiology of chronic heart disease and the long-term effects are still unknown, and the results of a multicentre Canadian study of continuous positive airway pressure in chronic left ventricular failure are therefore awaited [36]. The data presented in this study support the use of continuous positive airway pressure as an effective treatment for reducing sleep disordered breathing in patients with cardiac failure.

Acknowledgements. The authors thank A. Lux for the statistical evaluation of the data. The authors also thank Resmed UK for loaning two VPAP II flow generators for this study.

\section{References}

1. Javaheri S. Central sleep apnoea-hypopnoea syndrome in heart failure: Prevalence, impact, and treatment. Sleep 1996; 19: S229-S231.

2. Köhnlein T, Hoffmann B, Klein H, Welte T. CheyneStokes respiration during sleep in patients with low cardiac output due to chronic coronary artery disease or dilated cardiomyopathy. Eur Respir J 1998; 12: 124s. 
3. Naughton MT. Pathophysiology and treatment of Cheyne-Stokes respiration. Thorax 1998; 53: 514-518.

4. Köhnlein T, Welte T, Tan LB, Elliott MW. Central sleep apnoea syndrome in patients with chronic heart disease. A critical review of the current literature. Thorax 2002; 57: 547-554.

5. van de Borne P, Oren R, Abouassaly C, Anderson E, Somers VK. Effect of Cheyne-Stokes Respiration on muscle sympathetic nerve activity in severe congestive heart failure secondary to ischemic or idiopathic dilated cardiomyopathy. Am J Cardiol 1998; 81: 432-436.

6. Mann DL, Kent RL, Parson B, Cooper G. Adrenergic effects on the biology of the mammalian cardiocyte. Circulation 1992; 85: 790-804.

7. Lanfranchi PA, Braghiroli A, Bosimini E, et al. Prognostic value of nocturnal Cheyne-Stokes Respiration in chronic heart failure. Circulation 1999; 99: 1435-1440.

8. Hanly PJ, Zuberi-Khokhar NS. Increased mortality associated with Cheyne-Stokes respiration in patients with congestive heart failure. Am J Respir Crit Care Med 1996; 153: 272-276.

9. Andreas S, Hagenah G, Möller C, Werner GS, Kreuzer H. Cheyne-Stokes respiration and prognosis in congestive heart failure. Am J Cardiol 1996; 78: $1260-1264$.

10. Köhnlein T, Klante T, Elliott MW, Welte T. Heart failure and central respiratory dysregulation. CheyneStokes respiration during sleep in advanced left heart failure. Pneumologie 2001; 55: 13-20.

11. Naughton MT, Liu PP, Benard DC, Goldstein RS, Bradley TD. Treatment of congestive heart failure and Cheyne-Stokes respiration during sleep by continuous positive airway pressure. Am J Respir Crit Care Med 1995; 151: 92-97.

12. Guilleminault C, Stoohs R, Labanowski M, Simmons J, Clerk A. Cardiac failure, snoring, ventricular arrhythmias and nasal bilevel positive pressure ventilation. Sleep 1993; 16: S139-S140.

13. Calverley PM. Nasal CPAP in cardiac failure: case not proven. Sleep 1996; 19: S236-S239.

14. Buckle P, Millar T, Kryger MH. The effect of shortterm nasal CPAP on Cheyne-Stokes respiration in congestive heart failure. Chest 1992; 102: 31-35.

15. Task Force of the Working Group on Heart Failure of the European Society of Cardiology: The treatment of heart failure. Eur Heart $J$ 1997; 18: 736-753.

16. Liston R, Deegan PC, McCreery C, Costello R, Maurer B, McNicholas WT. Haemodynamic effects of nasal continuous positive airway pressure in severe congestive heart failure. Eur Respir J 1995; 8: 430-435.

17. Staniforth AD, Kinnear WJ, Starling R, Cowley AJ. Nocturnal desaturation in patients with stable heart failure. Heart 1998; 79: 394-399.

18. American Thoracic Society. Indications and standards for cardiopulmonary sleep studies. Am Rev Respir Dis 1989; 139: 559-568.

19. Rechtschaffen A, Kales A. A manual of standardised terminology, techniques and scoring system for sleep stages of human subjects. Washington DC, US Government Printing Office, 1968 (NIH publication No. 204).

20. Hall MJ, Xie A, Rutherford R, Ando SI, Floras JS, Bradley TD. Cycle length of periodic breathing in patients with and without heart failure. Am J Respir Crit Care Med 1996; 154: 376-381.

21. Johns MW. A new method for measuring daytime sleepiness: the Epworth sleepiness scale. Sleep 1991; 14: $540-545$.

22. Ware JE Jr, Sherbourne CD. The MOS 36-item shortform health survey (SF-36). I. Conceptual framework and item selection. Med Care 1992; 30: 473-483.

23. McHorney CA, Ware JE, Lu JFR, Sherbourne CD. The MOS item short-form health survey III. Tests of data quality scaling assumptions and reliability across diverse patient groups. Med Care 1994; 32: 40.

24. American Thoracic Society: Indications and standards for use of nasal continuous positive airway pressure (CPAP) in sleep apnea syndromes. Am J Respir Crit Care Med 1994; 150: 1738-1745.

25. Krachman SL, D'Alonzo GE, Berger TJ, Eisen HJ. Comparison of oxygen therapy with nasal continuous positive airway pressure on Cheyne-Stokes respiration during sleep in congestive heart failure. Chest 1999; 116: $1550-1557$.

26. Bradley TD, Holloway RM, McLaughlin PR, Ross BL, Walters J, Liu PP. Cardiac output response to continuous positive airway pressure in congestive heart failure. Am Rev Respir Dis 1992; 145: 377-382.

27. Naughton MT, Benard DC, Rutherford R, Bradley TD. Effect of continuous positive airway pressure on central sleep apnoea and nocturnal pCO2 in heart failure. Am J Respir Crit Care Med 1994; 150: 1598-1604.

28. Javaheri S. Effects of continuous positive airway pressure on sleep apnea and ventricular irritability in patients with heart failure. Circulation 2000; 101: 392-397.

29. Teschler H, Döhring J, Wang YM, Berthon-Jones M. Adaptive pressure support servo-ventilation. A novel treatment for Cheyne-Stokes respiration in heart failure. Am J Respir Crit Care Med 2001; 164: 614-619.

30. Becker $\mathrm{H}$, Grothe L, Ploch $\mathrm{T}$, et al. Intrathoracic pressure changes and cardiovascular effects induced by nCPAP and BiPAP in sleep apnoea patients. J Sleep Res 1995; 4: 125-129.

31. Sullivan CE, Grunstein RR. Continuous positive airway pressure in sleep-disordered breathing. In: Kryger $\mathrm{MH}$, Roth $\mathrm{T}$, Dement $\mathrm{WC}$, eds. Principles and Practice of Sleep Medicine, 2nd Edn. Philadelphia, WB Saunders 1994; pp. 694-705.

32. Naughton MT, Rahman MA, Hara K, Floras JS, Bradley TD. Effect of continuous positive airway pressure on intrathoracic and left ventricular transmural pressure in patients with congestive heart failure. Circulation 1995; 91: 1725-1731.

33. Mehta S, Liu PP, Fitzgerald FS, Allidina YK, Bradley TD. Effects of continuous positive airway pressure on cardiac volumes in patients with ischemic and dilated cardiomyopathy. Am J Respir Crit Care Med 2000; 161: 128-134.

34. Brambilla I, Micallef E, Sacerdoti C, Arlati S, Rolo J. Value of nocturnal monitoring of transcutaneous $\mathrm{O}_{2}$ and $\mathrm{CO}_{2}$ pressures in adults with respiratory failure. Respiration 1985; 48: 81-90.

35. Takasaki Y, Orr D, Popkin J, Rutherford R, Liu P, Bradley TD. Effect of nasal continuous positive airway pressure on sleep apnea in congestive heart failure. Am Rev Respir Dis 1989; 140: 1578-1584.

36. Bradley TD, Logan AG, Floras JS. Rationale and design of the Canadian continuous positive airway pressure trial for congestive heart failure patients with central sleep apnea - CANPAP. Can J Cardiol 2001; 17: $677-684$. 